\title{
Biochemical and clinical relevance of alpha lipoic acid: antioxidant and anti-inflammatory activity, molecular pathways and therapeutic potential
}

\author{
Daniele Tibullo $^{1} \cdot$ Giovanni Li Volti $^{1} \cdot$ Cesarina Giallongo $^{1} \cdot$ Sonia Grasso $^{1} \cdot$ \\ Daniele Tomassoni $^{2}$ - Carmelina Daniela Anfuso ${ }^{1}$. Gabriella Lupo ${ }^{1}$. \\ Francesco Amenta ${ }^{3} \cdot$ Roberto Avola $^{1}$ - Vincenzo Bramanti ${ }^{1,4}$
}

Received: 24 May 2017/Revised: 20 June 2017 / Accepted: 27 June 2017

(C) Springer International Publishing AG 2017

\begin{abstract}
Background The molecular nature of lipoic acid (LA) clarifies its capability of taking part to a variety of biochemical reactions where redox state is meaningful. The pivotal action of LA is the antioxidant activity due to its ability to scavenge and inactivate free radicals. Furthermore, LA has been shown to chelate toxic metals both directly and indirectly by its capability to enhance intracellular glutathione (GSH) levels. This last property is due to its ability to interact with GSH and recycle endogenous GSH. LA exhibits significant antioxidant activity protecting against oxidative damage in several diseases, including neurodegenerative disorders. Interestingly, LA is unique among natural antioxidants for its capability to satisfy a lot of requirements, making it a potentially highly effective therapeutic agent for many conditions related with oxidative damage. In particular, there are evidences showing that
\end{abstract}

Responsible Editor: John Di Battista.

Daniele Tibullo and Giovanni Li Volti contributed equally to this work.

Roberto Avola

ravola@unict.it

Section of Medical Biochemistry, Department of Biomedical and Biotechnological Sciences, University of Catania, Via S. Sofia, 89, 95123 Catania, Italy

2 School of Bioscience and Veterinary Medicine, University of Camerino, Camerino, Italy

3 School of Medicinal Sciences and Health Products, University of Camerino, Camerino, Italy

4 Division of Microbiology and Virology, Villa Sofia Hospital, A.O.O.R. "Villa Sofia-Cervello", P.zza Salerno 1, 90146 Palermo, Italy

LA has therapeutic activity in lowering glucose levels in diabetic conditions. Similarly, LA supplementation has multiple beneficial effects on the regression of the mitochondrial function and on oxidative stress associated with several diseases and aging.

Aim The aim of the present review is to describe the molecular mechanisms underlying the beneficial effects of LA under various experimental conditions and disease and how to exploit such effect for clinical purposes.

Conclusion LA has pleiotropic effects in different pathways related with several diseases, its use as a potential therapeutic agent is very promising.

Keywords Alpha lipoic acid - Antioxidant - Chelation · Free radical scavenger - Glutathione - Inflammation . Therapeutic potential

$\begin{array}{ll}\text { Abbreviations } & \\ \text { LA } & \text { Lipoic acid } \\ \text { DHLA } & \text { Dihydrolipoic acid } \\ \text { R-LA or (+)LA } & \text { R-enantiomer lipoic acid } \\ \text { S-LA or (-)LA } & \text { S-enantiomer lipoic acid } \\ ( \pm) \text { LA } & \text { Raceme lipoic acid } \\ \text { ROS } & \text { Reactive oxygen species } \\ \text { RNS } & \text { Reactive nitrogen species } \\ \text { SOD } & \text { Superoxide dismutase } \\ \text { GSH } & \text { Glutathione } \\ \text { GSSG } & \text { Disulfide form of glutathione } \\ \text { MAPK } & \text { Mitogen activated protein Kinases } \\ \text { PI3-K } & \text { Phosphatidyl inositide 3-kinase } \\ \alpha G P C & \text { L- } \alpha \text {-glycerylphosphorylcholine } \\ \text { GPCR } & \text { G protein coupled receptor } \\ \text { ERK } & \text { Extracellular regulated Kinases } \\ \text { JNK } & \text { c-Jun N-terminal kinase }\end{array}$

Abbreviations

LA

DHLA

R-LA or $(+)$ LA

S-LA or $(-)$ LA

$( \pm)$ LA

ROS

RNS

SOD

GSH

GSSG

MAPK

PI3-K

$\alpha \mathrm{GPC}$

JNK
Lipoic acid

Dihydrolipoic acid

R-enantiomer lipoic acid

S-enantiomer lipoic acid

Raceme lipoic acid

Reactive oxygen species

Reactive nitrogen species

Superoxide dismutase

Glutathione

Disulfide form of glutathione

Mitogen activated protein Kinases

Phosphatidyl inositide 3-kinase

L- $\alpha$-glycerylphosphorylcholine

$\mathrm{G}$ protein coupled receptor

c-Jun $\mathrm{N}$-terminal kinase 
AKT

NF-kB

IGF-1

IR

IRS1

IRS-1

AMPK

PTP1B

LKB-1

CaMKK

PGC-1-alpha

EAE

$\mathrm{AD}$

AChE

AGEs

HNE

PKC

GLUT
The protein kinase $\mathrm{B}$

Nuclear factor-KB

Insulin-like growth factor-1

Insulin receptor

Insulin receptor substrate 1

Insulin receptor substrate-1

5' Adenosine monophosphate-activated protein kinase

Cellular protein tyrosine phosphatases

Liver kinase B1

$\mathrm{Ca} /$ calmodulin dependent protein kinase

Proliferator activated receptor-gamma coactivator-1alpha

Experimental

encephalomyelitis

autoimmune

Alzheimer's disease

Acetylcholinesterase

Advanced glycation end product

4-Hydroxy-2-nonenal

Protein kinase C

Glucose transport protein

\section{Introduction}

\section{Biochemical properties of lipoic acid}

Lipoic acid (1,2-dithiolane-3-pentanoic acid; LA) as well as its reduced form dihydrolipoic acid (DHLA) are compounds having a chiral center (Fig. 1). LA contains two thiol groups, which may be oxidized or reduced. It is part of a redox pair, being the oxidized member of the reduced form dihydrolipoic acid (DHLA) and both the oxidized and reduced forms of LA are antioxidants. In addition, the
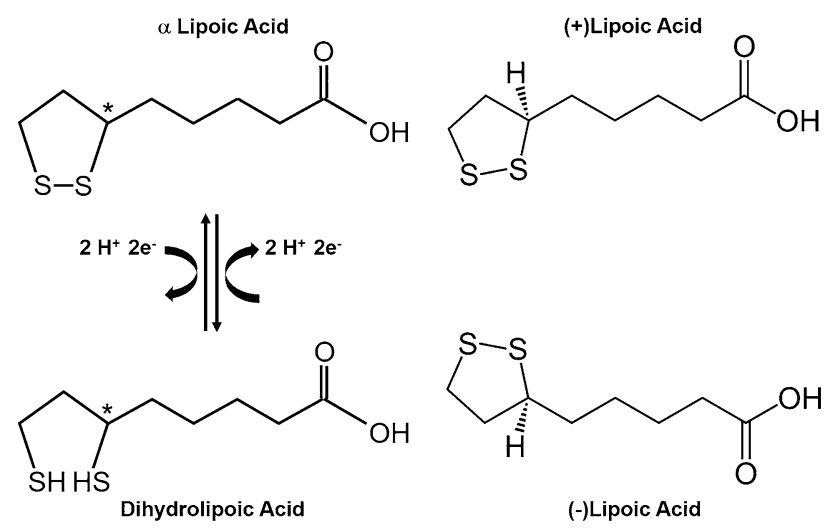

Fig. 1 Lipoic acid structure: lipoic acid (1,2-dithiolane-3-pentanoic acid) is an eight-carbon dithiol compound with a high reduction potential. It has one chiral center that is denoted by an asterisk

asymmetrical carbon atom, provides special optical properties.

LA has two enantiomers: the R-enantiomer [R-LA or $(+) \mathrm{LA}$ ] and the S-enantiomer [S-LA or (-) LA]. LA is present in nature as R-enantiomer (Fig. 1), but synthetic LA is a racemic mixture of $(+) \mathrm{LA}$ and $(-) \mathrm{LA}[(+/-) \mathrm{LA}]$. Furthermore, both forms have different functions. LA is also a pivotal component of mitochondrial complex of four important proteins participating in the synthesis and degradation of glycine molecule. LA is also a cofactor for several enzymes including pyruvate dehydrogenase complex (PDC) and $\alpha$-ketoglutarate dehydrogenase complex (KDC), two mitochondrial enzymes involved in glucose metabolism and energy production [27].

In view of its important role in biochemical processes, LA was enclosed into vitamin B complex, although, now researchers do not consider it a vitamin. The chemical activity of LA and DHLA is mainly based in its dithiolane ring (Fig. 1), in addition, the position of the two sulfur atoms in the ring creates an exceptionally high electron density, which confers special properties to LA. It is well known that in biological systems only the NAD $(\mathrm{P}) \mathrm{H} /$ $\mathrm{NAD}(\mathrm{P})+$ redox couple has a higher reduction potential. DHLA - the reduced form of LA — exercises an antioxidant effect directly by donating electrons to a pro-oxidant or an oxidized molecule. It can regenerate reduced vitamin $\mathrm{C}$ from dehydroascorbic acid and it can indirectly regenerate vitamin $\mathrm{E}$ back from its oxidized state. Moreover, LA metabolites have been shown to have anti-inflammatory and antioxidant effects [37]. However, it has been shown that LA is able to exert a significant antioxidant effect through a scavenger activity on free radicals [52], as well as its capability of LA to chelate metals [19]. Thus, the chemical nature of LA and DHLA make them capable of taking part to a variety of biochemical reactions where redox state is meaningful.

A significant activity of LA is the capability to chelate toxic metals and also to increase glutathione levels inside the cells. Glutathione plays important role in the capability of the system to chelate and discharge a wide variety of toxins and toxic metals. Several metals known to form these complexes are manganese, zinc, cadmium, lead, cobalt, nickel, iron, copper, cadmium, arsenic and mercury.

\section{Antioxidant properties of lipoic acid}

\section{Oxidative damage: ROS-RNOS}

It is well known that oxidative stress is implicated in the development and evolution of a lot of diseases and there are many factors related to oxidative stress as well as to the development of several pathologies. Reactive oxygen 
species (ROS) are reactive molecules derived from the natural byproduct of the oxygen metabolism and having significant roles in the homeostasis and in the cell-signaling. Nitric oxide (NO) is a soluble and highly diffusible gas generated by a wide variety of cell types including vascular endothelial cells [27]. Excess production of endogenous NO under inflammatory conditions can be toxic to vascular endothelial cells [54]. Unregulated NO production leads to an increase in reactive nitrogen species (RNS) resulting in nitrosative stress (NS), which may give rise to significant pathological outcomes including cellular injury and disease. Specifically, NS is involved in the pathogenesis of low-grade chronic inflammatory-associated diseases such as atherosclerosis and type 2 diabetes. A better understanding of NS-associated underlying mechanisms may help improve therapeutic outcomes for these diseases [9]. Cell metabolism produces other free radicals from nitrogen species, called reactive nitrogen species (RNS). ROS and RNS during physiological conditions have been demonstrated to be able to mediate a variety of meaningful functions, such as regulation of signal transduction, induction of mitogenic response, the involvement on immune response, etc. ROS are balanced through the presence of antioxidant groups to take their level invariable in organisms. These antioxidant systems can be both enzymatic and non-enzymatic. Breaking the above mentioned balance through the over production of ROS as well as through the reduction of antioxidants, can be deleterious and it conducts to oxidative stress. Under these conditions, the unbridled production of free radical induces damage on cell membrane via lipid peroxidation, modifying signal and structural proteins to drive to misfolding and aggregation, and oxidizing RNA/DNA to arrest the transcription and provoke gene mutation. The bad consequences of the reactive oxygen species on the cells are: lipid peroxidation, DNA damage, aminoacids oxidations, etc.

When oxidative stress happens, cells reply to neutralize the oxidant effects and to restore redox balance by resetting critical homeostatic parameters. This cellular activity induces the activation or the silencing of genes encoding defensive enzymes, transcription factors and structural proteins. According to the free radical theory of aging, oxidative stress enhances with increasing age, this condition brings to cellular dysfunction by accumulation of oxidation products of lipids, nucleic acids, proteins, etc. (Fig. 2). When the balance between the production of ROS and its removal is modified and altered, the cells suffer oxidative stress. In this field, lipoic acid (LA) has stimulated increasing interest for its the antioxidant and therapeutic potential.

\section{Mechanisms of protection from oxidative damage}

The cells possess innate mechanisms capable to fight free radicals, neutralizing the consequences and slowing down the processes of cellular modification and aging. The two main mechanisms of protection are:

- to remove free radicals through enzymatic activities: the mitochondrial or cytoplasmic enzymes are "scavengers", such as superoxide dismutase (SOD).

- to interact with free radicals giving them the missing electron and becoming less active as a result of the antioxidant agents' activity (vitamins E, A, C, selenium, zinc, glutathione).

Antioxidants are not a homogeneous category of substances, in fact, they are vitamins, minerals, essential amino acids. Antioxidants may operate individually or cooperate protecting each other when they are oxidized. In addition, each antioxidant is highly specific and can only interact with one or two free radicals.

It is well known that among the antioxidants of the latest generation, lipoic acid and DHLA are capable to fulfill the function of "scavenger" of hydroxyl radicals, hypochlorous acid, of oxygen singlet, and of peroxyl radicals. Furthermore, they are both chelating iron, copper and other transition metals responsible for the oxidation of many substances. Alpha lipoic acid binds these metals to constitute stable complexes. Furthermore, alpha lipoic acid is capable to prevent the oxidation of ascorbic acid catalyzed by copper and inhibit lipid peroxidation catalyzed by copper. In addition, DHLA seems to be capable to regenerate other antioxidants such as vitamin $\mathrm{E}$ and ascorbic acid, by their radical forms. As is known that, vitamin $\mathrm{E}$ is the main antioxidant that preserves the membranes which are the main target of the lipid peroxidation induced by free radicals.

However, although the peroxyl radicals can be quickly produced in the membranes, the harmful oxidation of membrane lipids does not happen as a rule, and the vitamin $\mathrm{E}$ is not depleted rapidly, leading subsequently to a deficiency of vitamin $\mathrm{E}$.

This evident contradiction can be clarified by the hypothesis of "recycling" of vitamin E, where its antioxidant ability is steadily restored by other antioxidants. Antioxidants that recover vitamin $\mathrm{E}$ are vitamin $\mathrm{C}$, the ubiquinones and thiols. Furthermore, DHLA protects cells from lipid peroxidation only in the presence of vitamin $\mathrm{E}$ or directly reducing the tocopheryl radical, that is, the vitamin $\mathrm{E}$ in the radical form, or reducing other antioxidants (such as ascorbic acid) which subsequently regenerate vitamin $\mathrm{E}$. 
Fig. 2 Reactive oxygen species (ROS) generated by endogenous as well as exogenous sources, cause damage and accumulation of proteins, lipids and DNAs, when defensive (repair) mechanisms of body become weak. These ROS also modulate the signal transduction pathways. These disturbances cause organelle damage, changes in gene expression followed by altered cellular responses which ultimately results into aging

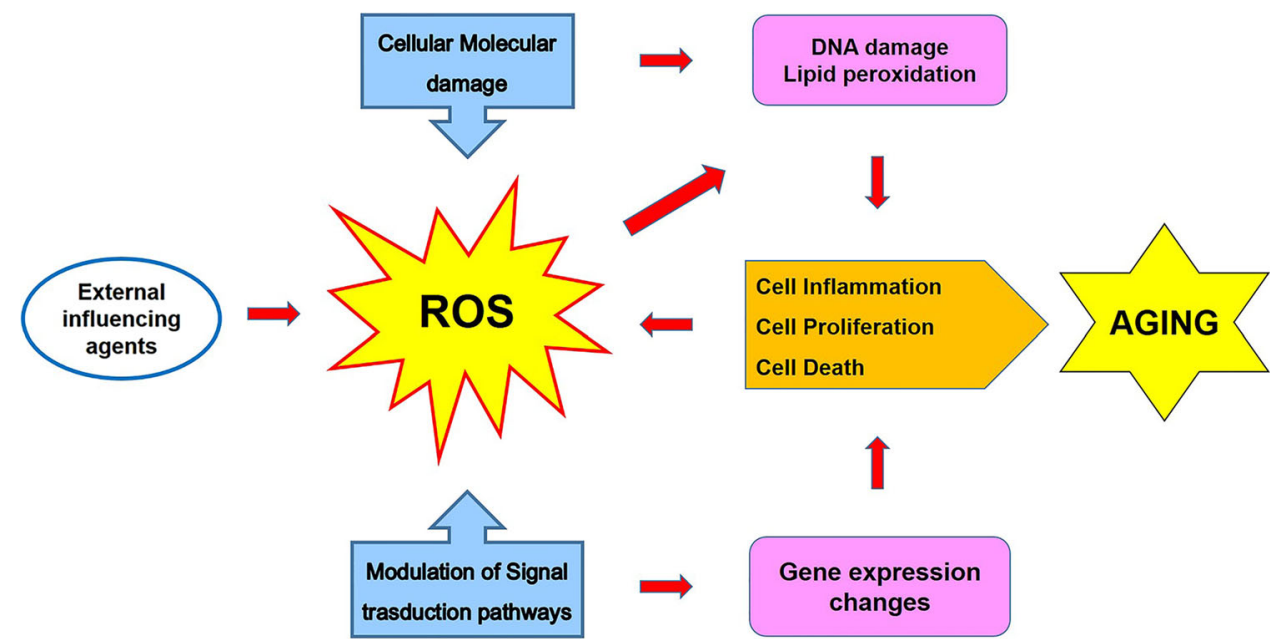

\section{Chelation of metals}

Under physiological conditions, metal ions such as iron $(\mathrm{Fe})$, zinc $(\mathrm{Zn})$ and copper $(\mathrm{Cu})$ are meaningful and indispensable cofactors for normal function of some proteins. The lack or excess of transition metal, however, can conduct to the progression of several diseases. $\mathrm{Zn}^{2+}$ can cause oxidative stress and mediate neuronal toxicity in several neurological disorders; $\mathrm{Cu}$ and $\mathrm{Fe}$ also have the ability to take part in the production of free radicals under pathological conditions. Both LA and DHLA well demonstrated to be able to chelate metal ions by constituting stable complexes with the ions. Furthermore, LA was also able to inhibit liposomal peroxidation $\mathrm{Cu}^{2+}$ catalyzed [50]. The relevant chelating capability of LA and DHLA is due to the arrangement of charges on the fatty acid-like backbone that allows to envelop copper and iron ions [71]. Regardless of the mechanism, it is clear that LA possesses a meaningful therapeutic potential in diseases in which the transition metals mediated cellular toxicity.

In addition, LA and DHLA are also implicated in heavy metal detoxification. Exposure to heavy metals such as mercury, lead, cadmium and arsenic has become an increasingly recognized source of illness worldwide due to rising levels in air, water and food. Thus, there is growing interest in locating and utilizing agents that are capable to chelate these toxic metals for therapeutics strategies [62].

\section{Lipoic acid-glutathione interaction}

Glutathione (GSH) is an antioxidant capable to prevent and avoid injuries and damages to several cellular components, provoked by reactive oxygen species, such as free radicals, heavy metals and lipid peroxides. In normal conditions the $90 \%$ of the total glutathione stock exists in the reduced form (GSH) and the $10 \%$ exists in the disulfide form
(GSSG). The relationship of reduced glutathione and oxidized glutathione inside the cells is frequently used as a measurement of oxidative stress as well as cellular toxicity.

Glutathione possesses manifold functions, among which to be the main endogenous antioxidant produced by the cells, participating directly to the neutralization of free radicals and reactive oxygen species, as well as preserving exogenous antioxidants such as vitamins $\mathrm{C}$ and $\mathrm{E}$ in the reduced forms. Another main function of glutathione is to regulate the nitric oxide cycle. Furthermore, the glutathione participates to several metabolic and biochemical reactions, such as DNA synthesis and repair, protein synthesis, amino acid transport and enzyme activation. Moreover, it has an essential function in iron metabolism. Therefore, the glutathione activity influences every system in the organism, particularly the immune system and the nervous system.

A main property of LA is its interaction with GSH and its ability to recycle endogenous GSH also. In old rats where GSH levels were reduced, the intravenous administration of LA ( $40 \mathrm{mg} / \mathrm{kg}$ body weight) restored tissue levels of GSH in both the heart and brain [70]. Equally, LA also enhances GSH levels in T cells, human erythrocytes, glial cells and lymphocytes [55].

The regeneration of GSH is due to the capability of DHLA to recycle GSH from GSSG. In addition to generating GSH by reducing GSSG, LA may also be involved in the glutathione synthesis. The oxidation-reduction process of LA to DHLA is an uninterrupted source of cysteine, which is the rate-limiting amino acid for glutathione production [55]. Therefore, the ability of LA to regenerate GSH is a main mechanism, in addition to being able to scavenge free radicals and chelate metal ions that may better clarify its therapeutic efficacy in several pathologies.

Some interesting researches shown that the $(+)$ LA is more powerful than (-)LA in its ability to stimulate 
glucose uptake in L6 myotubes [14], as well as to enhance insulin-stimulated glucose uptake in obese Zucker rats [32]. On the other hand, the S-enantiomer exerts a slightly increased affinity for glutathione reductase [57], thus the two forms of LA differ in their ability to exercise the various biological activities.

LA is unique among natural antioxidants in its ability to fulfill a lot of requirements, making it a potentially highly effective therapeutic agent for a number of pathological conditions implicated with oxidative damage.

Summarizing, the antioxidant capacities of LA: to scavenge reactive oxygen species (ROS); to restore endogenous antioxidants, such as glutathione, vitamins $\mathrm{E}$ and $\mathrm{C}$; the metal chelating activity, resulting in reduced ROS production. Some researches showed that LA protects against oxidative injury in various disease processes, including neurodegenerative disorders $[15,53]$. Despite the capability of LA to be "a scavenger of ROS" appears to be responsible, at least partially, of its neuroprotective effects, it remains unknown whether the neuroprotective effects of LA may also occur through other mechanisms, such as induction of the endogenous antioxidants and phase 2 enzymes in neuronal cells. Moreover, the increasing endogenous defenses might provide defense against oxidative/electrophilic neuronal cell injury.

Certain researches showed the neuroprotective effects of LA in neuronal cells. The neuroprotection occurs after extended pretreatment with LA and is probably due to the free radical scavenger properties of DHLA endogenously formed [46]. Some interesting researches assessed the effect of LA treatment on the expression of some proliferation and differentiation biomarkers of astroglial cells cultures [6]. The findings highlight the antioxidant and neuroprotective role played by the $(+) \mathrm{LA}$, indicating the modulation of biochemical processes correlated with the proliferation and differentiation of astrocyte cultures [6]. In particular, the crucial role played by the $(+)$ LA especially after acute treatment of $24 \mathrm{~h}$ in their in vitro model, denoted that this antioxidant and neuroprotective drug, operates particularly in proliferating and differentiating astroglial cell in cultures, as well as, during signaling transduction mechanism [16].

However, it is also well known that LA mimics insulin by stimulating glucose uptake in several cell types, including nerve cells [16]. It is also reported that LA does not bind to the extracellular domain of the insulin receptor, but to the tyrosine kinase domain inside the cell. In addition, LA also modulates MAPK, PI3-K and NFk activities, which may be independent respect to their activation by receptor tyrosine kinases [62]. The antioxidant role exercised by LA and its capability in restoring glutathione content may be related to proliferative and differentiative state of astrocyte cells, as showed by up and down modulation of astroglial biomarkers expression assessed [16].

This finding underscores the neuroprotective action against oxidative stress stimulated by LA.

In addition, since LA exerts a main role as antioxidant and metabolic component of some enzymatic complexes involved in glucose metabolism of different cell types, it is interesting to relate the results obtained by another research study [22] in which the authors investigated the effect of both LA alone or in combination with $\mathrm{L}-\alpha$-glycerylphosphorylcholine $(\alpha \mathrm{GPC})$ on astroglial cell proliferation and differentiation in cultures. The results show that the neuroprotective action against oxidative stress mimicked by LA and $\alpha \mathrm{GPC}$. The results show that the neuroprotective action versus oxidative stress induced by LA and $\alpha$ GPC. These discoveries may be exploited to better clarify the antioxidant and metabolic activity played by LA during astroglial cell proliferation and differentiation, as well as during an interactive cross-talk among glial and neuronal cells, after brain injuries or damage correlated with the oxidative stress.

\section{Molecular pathways}

\section{Cellular signaling pathways}

A lot of signaling cascades are implicated in oxidative stress and in the inflammatory reactions. The tyrosine kinase signaling cascades are activated by various transmembrane receptors conducting to the pathogenesis of allergic airway inflammation [82]. G protein coupled receptor (GPCR) signaling moderates responses throughout the immune, nervous, and muscular systems that might collaborate to the pathogenesis of allergic processes.

The ERK, p38 MAP kinase, JNK, AKT and NF-kB signaling cascades, control the oxidative stress response, the inflammatory gene expression, the proliferation and cell survival $[18,25,66]$.

In addition, it is well known that the capability of LA is to activate various signal transduction pathways and switch on some transcription factors, indicating that the internalization of LA may be also mediated by ligand-receptor binding.

\section{Lipoic acid and insulin/IR/Akt pathway}

LA performs a lot of functions in the insulin metabolic pathways, glucose uptake and glycogen synthesis with several differences between both isomers. For example, it is well known that R-LA increments the translocation of GLUT1 and GLUT4 to the plasmatic membrane of adipocytes [44, 67]. Furthermore, these happenings were 
related to the enhanced activity of some proteins from the insulin signaling pathway, as the insulin receptor (IR), the insulin receptor substrate 1 (IRS1), the phosphatidyl inositide 3 kinase (PI3K) and the protein kinase B (AKT). These occurrences were also observed with some animal models of insulin-resistance, the known Zucker rats. There is still a debate in progress if this activity of LA is fully insulin dependent or not [50, 67]. Moreover, in rat animal model, LA forestalled the glycogen synthesis and this action is considered as a pro-oxidant effect [48].

A lot of genes implied in the mediation of cellular stress resistance are related to the insulin signaling pathway. LA is similar to the insulin in its activity able to switch on signaling molecules in the insulin/insulin-like growth factor-1 (IGF-1) pathway.

A very important enzyme that plays a key role in this field is the protein kinase $\mathrm{B} / \mathrm{Akt}$, that is switched on by the insulin/IGF-1 pathway.

Anyway, the interaction between LA and the insulin signaling pathway is now well accepted [15], even if it is not so obvious what proteins are targets of LA action. Some research findings in a preadipocyte cell culture, demonstrated that although Akt became phosphorylated in response to LA within $30 \mathrm{~min}$, the IR and insulin receptor substrate-1 (IRS-1) protein were not phosphorylated [8].

However, in adipocytes cell cultures, it was demonstrated that IR was indeed phosphorylated after LA treatment, and this was specific to LA and not DHLA [44]. Others researchers also showed that both IRS-1and PI3K were phosphorylated after following LA treatment [85].

\section{Lipoic acid and phosphatases}

Beyond to stimulate the IR, LA seems to be able to stop the cellular protein tyrosine phosphatases (PTP1B), thereby interfering with the otherwise inhibitory dephosphorylation of the IR tyrosine kinase domain [8]. The observed inhibition of PTP1B with $\mathrm{H}_{2} \mathrm{O}_{2}$ and LA overlapped with decreased sulfhydryl content, still suggesting that LA either directly modified critical thiol groups on this phosphatase or induced oxidants that did the same. Other phosphatases may be stopped by LA, which include protein phosphatase$2 \mathrm{~A}$, an important serine/threonine enzyme connected with the insulin-mediated and other signaling processes [68].

Particularly, there is a growing evidence that (DH)LA, via its redox nature, modulates significant signal transduction pathways that increase the endogenous cellular antioxidants (e.g., GSH), lower inflammation (e.g., NF$\mathrm{kB}$ ), and enhance the cell survival opportunities (e.g., IR/ Akt) in times of damage. Induction of kinases and transcription factors or the inhibition of phosphatases include classic examples of signal amplification that would have longer term effects than if LA operated only as a transient oxidant scavenger. Furthermore, basal concentrations of LA could influence cell-signaling systems, which is consistent with the limited storage of LA in tissues following its oral administration.

\section{Lipoic acid and nuclear factor kappa B}

Lipoic acid has both antioxidant and anti-inflammatory effects. It is well known that the antioxidant effects are due to increased antioxidant enzymes, such as glutathione reductase, restoration of the reduced/oxidized glutathione ratio, and reduction of $\mathrm{NADP}^{+}[7]$.

Oxidative stress associated to inflammation determines early vascular events correlated to atherogenesis, including the up modulation of vascular adhesion molecules. These events require the activation of NF-kB, a transcription factor inducing the expression of a lot of genes implicated in the inflammatory processes and endothelial cell migration. The ROS-mediated inflammation induces activation of nuclear factor kappa B (NF- $\mathrm{kB}$ ), activator protein 1 (AP1 ), and mitogen-activated protein kinases (MAPK). NF- $\kappa B$ at the same time facilitates the production of inflammatory cytokines: tumoral necrosis factor alpha (TNF- $\alpha$ ), interleukin-(IL-) 6, cyclooxygenase 2 (COX-2), and inducible nitric oxide synthase (iNOS) [60]. Nuclear factor kappa B (NF-kB) is stored in an inactive form in the cytosol because of its capacity in binding to an inhibitor kinase of NF-kB activity, IKK [85]. Oxidative stress is also correlated to hyperglycemia $[13,14,26,84]$ and the existence of other conditions such as viral infections, pathogens and radiation are believed to phosphorylate IkB resulting in its degradation, release and activation of NF-kB. This last one moves into the nucleus to induce the transcription of several molecules correlated to inflammation, vascular adhesion and migration of monocytes [85].

LA inhibits NF-kB [51] probably because avoid the degradation of IkB through modulation of upstream kinases like MAPK [52] or owing to its power to regenerate vitamin $E$ resulting in inhibition of protein kinase $C$ which is also able to phosphorylate IkB [4]. This last activity of LA seems to be independent of its antioxidant action [86]. Therefore, the anti-inflammatory effects of LA are due to suppression of inflammatory activity from NF- $\kappa \mathrm{B}, \mathrm{TNF}-\alpha$ and IL- 6 and increased anti-inflammatory proteins, such as nuclear erythroid 2- related factor (Nrf2) [45].

\section{Lipoic acid and adenosine monophosphatase protein kinase}

LA performs several significative functions in the activity and expression of $5^{\prime}$ adenosine monophosphate-activated protein kinase (AMPK) in peripheral tissues and in brain (hypothalamus). AMPK is considered a multifunctional 
protein associated with a lot of intracellular pathways correlated to metabolism, stress response, cell cycle and aging [69]. Actually, AMPK performs an important role in connecting nutritional factors and cancer and is considered as a hopeful therapeutic target for cancer prevention and treatment [88].

AMPK is an energy sensor switched on by liver kinase B1 (LKB-1) and $\mathrm{Ca} / \mathrm{calmodulin}$ dependent protein kinase (CaMKK). Activation of AMPK arised by down regulation of transcriptional events that promote synthesis of gluconeogenic enzymes, synthesis of fatty acids and up regulation of metabolic pathways, resulting in ATP production through glucose and fatty acids oxidation [88]. Moreover, AMPK can elicit the translocation of GLUT4 to plasma membrane independent of insulin action [88]. It is still unknown about the mechanism inducing the activation of AMPK by LA in peripheral tissues yet [21]. Interesting evidences on the myoblast cells in culture shows that LA could increase the activation of AMPK indirectly by the activation of CaMKK in turn induced by an increment of $\mathrm{Ca}^{2+}$ [65]. In fact, the inhibition of CaMKK removes this last action [65]. Indeed, these actions of LA-AMPK could also enhance energy expenditure by increasing the activity of protein kinaseperoxisome proliferator activated receptor-gamma coactivator-1alpha (PGC-1-alpha) signaling pathway. This last one is accountable of mitochondrial biogenesis [79]. Every mentioned behaviors resulting from the activation of AMPK will provoke a reduction of plasma glucose, an increment of insulin sensitivity and probably weight loss [69].

Moreover, it has been widely demonstrated the action of LA on AMPK in insulinoma cells culture and Langerhans islets. The results of present research study showed a decrement of insulin secretion by $25-30 \%$ at low as well as at high glucose concentrations [72]. This last evidence was observed and confirmed with acute and chronic treatment of LA. Another interesting study showed a protective effect of LA on 2-deoxy-D-ribose that caused oxidative damage and insulin expression in cell culture [35]. This protective activity seems to be correlated to an enhanced intracellular GSH level. Both studies have showed important details about the multiple effects of LA upon beta cells. The study design and the concentration of LA performed in these experiments could be correlated to the different results obtained [35]. It is also well known that LA is also capable to modulate the activity of AMPK in the brain through metabolic pathways that forbid ATP production such as oxidative stress, loss of glucose as well as ischemia and hypoxia [59]. Several studies well reported that hypothalamic AMPK has an important role in the central regulation of appetite and energy metabolism, as well as reported that LA performs anti-obesity activity via down regulation of hypothalamic AMPK activity [34].

\section{Therapeutic potential of lipoic acid}

Some pathologies of nervous system, such as Alzheimer's disease and diabetic polyneuropathy, share common characteristics: inflammation and oxidative stress. The inflammatory process related with these pathologies can stimulate the overproduction of ROS and NOS. For instance, oxygen and nitrogen radicals generated by macrophages have been involved as intermediary of the demyelination and axonal injury in multiple sclerosis (MS) and its animal model, experimental autoimmune encephalomyelitis (EAE) [5, 36, 77]. In addition, studies of oxidative damage show enhancement of lipid peroxidation, protein and RNA oxidation in neocortical brain regions in late-stage Alzheimer's disease [39].

Furthermore, since oxidative stress cooperates to develop the disease pathogenesis, it has been investigated to use LA as a treatment alternative for Alzheimer's disease and diabetic polyneuropathy. There is evidence that LA performs therapeutic activity in diabetic condition because it is able to induce a lowering glucose levels. In fact, LA is administered to the patients suffering from diabetic polyneuropathy-a pathologic condition associated with increased oxidative stress [20, 76, 91].

Since LA stabilizes the major intracellular antioxidant system [87], its administration has multiple beneficial effects on the regression of the mitochondrial function and on oxidative stress associated with several diseases and aging. However, appropriate plasma levels need to be obtained to warrant maximum therapeutic benefit. The use of the LA as drug or food supplement is interfered by its rapid metabolism (man plasma half live of $30 \mathrm{~min}$ and bioavailability after oral administration of $30 \%$ ) and its stability problems since it is known that LA can polymerize. Its degradation in the presence of light was characterized by a physical change in the compound and a shift in the ultraviolet spectrum.

\section{Alzhèimer's disease}

Alzhèimer's disease (AD) is a chronic neurodegenerative disease characterized by the deposition of extracellular neuritic, $\beta$-amyloid peptide containing senile plaques in the hippocampal area and in the cerebral cortical districts [42]. It is characterized by deprivation of neurons and subsequent synaptic damage in the cerebral cortex and subcortical districts. This damage provokes complete atrophy and cell degeneration of the affected districts.

The symptoms of inflammation are also clear and evident around the senile plaques. These plaques start a cascade of uncontrollable and pathological changes 
conducting to increased levels of extracellular glutamate, that is in part responsible of the neuronal cell death [80].

At present, the patients affected by mild or moderate $\mathrm{AD}$, are mainly treated with symptomatic drugs such as acetylcholinesterase (AChE) inhibitors (donepezil, rivastigmine and galanthamine) [28]. These drugs reduce the rapidity at which acetylcholine is broken down and interact to enhance the concentration of $\mathrm{ACh}$ in the brain.

Due to its antioxidant properties and its capability to modulate various signaling cascades, LA is used as alternative therapy for AD. Some investigations "in vitro and in vivo" provided promising results. In fact, it was demonstrated that LA represses the advanced glycation end product (AGEs) induced by lipid peroxidation in neuronal cell line in vitro [17]. hippocampal neurons in primary culture were also protected and preserved by DHLA against amyloid beta-peptide and $\mathrm{Fe} / \mathrm{H}_{2} \mathrm{O}_{2}$ induced toxicity [40]. 4-Hydroxy-2-nonenal (HNE) is a highly reactive product of lipid peroxidation of unsaturated lipids that induces oxidative toxicity and conducts to neurodegeneration. Pretreatment of cortical neurons with LA before HNE attack caused an enhancement of neuronal cell survival, an increment of GSH and heat shock protein levels, as well as the activation of the pro-survival PI3 kinase, PKC and ERK1/2 signaling pathways [1]. Some researchers demonstrated that $1 \mu \mathrm{M} \alpha$-lipoate protected rat hippocampal neurons against glutamate induced cell damage [46]. In vivo studies in old rats fed a diet supplemented with LA for 2 weeks induced a development of mitochondrial function, an increment of metabolic rate and a decrement of oxidative damage [24]. In Tg2576 mice, a transgenic model of cerebral amyloidosis with $\mathrm{AD}$, the LA uptake improved learning and preservation of memory [58]. These research findings demonstrate the practicability of supplementation of lipoic acid to improve the treatment of AD symptoms. Another interesting study [83] demonstrated that $A \beta$ peptides could significantly inhibit the proliferation of $\mathrm{C} 6$ cells, while LA could reverse the inhibition effect of $A \beta$ and promote the recovery of cell viability. $A \beta$ exposure was also found to decrease the ratio of GSH and GSSH, and it reduced the expression of both MnSOD mRNA and protein in the mitochondria of C6 cells. However, LA pretreatment of A $\beta$-treated C6 glioma cells could reverse the expression changes seen in GSH and GSSH, increase the ratio of GSH and GSSH, and increase the expression levels of MnSOD mRNA and protein, restoring these to approximately control levels. In summary, the results of the study suggested that $A \beta$ exposure injured C6 cells through oxidative stress and oxidative damage in the mitochondria, which effect could be reversed by LA treatment, thereby further suggesting that LA might protect $\mathrm{A} \beta$-treated $\mathrm{C} 6$ cells by alleviating oxidative damage. These results confirmed the neuroprotective effect of LA and provided evidence of its mechanism; LA thus might provide a new treatment choice for neurological degenerative diseases.

\section{Diabetic neuropathy}

Diabetic neuropathy (DN) is a frequent and serious complication of both type 1 (DM1) and type 2 (DM2) diabetes. Diabetic neuropathy is a peripheral nerve dysfunction [60] as well as a progressive, debilitating condition with a major impact on patient morbidity, mortality, and quality of life [56]. DN is a diabetes complication that concerns sensory, autonomic and motor neurons of the peripheral nervous system, and it is also the higher source of death rate in diabetic patients [78]. The first risk factor for DN is hyperglycemia [64], but the pathology is assigned to oxidative stress, to the development of advanced glycation end products, to the uncontrolled activation of protein kinase $\mathrm{C}(\mathrm{PKC})$, that induce an increment of glucose flux by the polyol pathway and inflammation [11].

Some studies indicated that the enhanced glycemic control in diabetic patients reduces the risk of diabetic neuropathy as well as other complications [11].

LA has been administered in patients affected by DN in Germany for many years (over 30 years) [90]. In the rat model of streptozotocin-DN-induced, the intraperitoneal administration of LA improved nerve blood flow as well as the GSH levels [47].

An interesting study showed that lipid peroxidation was reduced in both the brain and sciatic nerve [49]. Another interesting study demonstrated that the treatment with $10 \mathrm{mg}$ LA per kilogram of body weight in some diabetic rabbits induced a significant reduction of serum glucose levels $[38,81]$. In addition, diabetes induced by reduction of GSH/GSSG ratio was attenuated and the accumulation of hydroxyl free radicals was abolished. These studies give strong and hopeful evidence for the therapeutic potential of LA in treating symptoms of diabetes and DN.

While much of the work on LA has used animal models to define its function, there have also been several important trials that examine the potential health benefits of LA. A lot of outcomes concerning the therapeutic administration of LA in humans lend support to its general role as antioxidant drug. The best trial for the administration of LA derived comes from studies on type 2 diabetes [15].

A lot of clinical trials of LA indicated that its oral or intravenous administration enhances nerve conduction rate and neuropathic symptoms, such as pain, burning, paresthesia and numbness $[3,89]$. To date, the therapeutic use of LA in the treatment of diabetic polyneuropathies represents the best documented and the most significant benefit of LA to human health. Furthermore, several experimental evidences support a role for LA as a mitigator of oxidative 
stress in this disease but also for its capability to influence glucose handling [47]. A number of reports show that LA improves glucose disposal in diabetic type 2 patients after administration of LA [30]. Others animal studies show that LA improves and enhance skeletal muscle glucose uptake as well as glucose tolerance in the whole-body and it is also helpful against insulin-resistance [29,73]. This improved glycemic treatment induced by LA may be due by its interaction with regulatory components of the insulin signaling cascade. In fact, LA stimulates the recruitment of glucose transport protein 4 (GLUT4) since its storage site in the golgi site towards the sarcolemma. This improves the glucose uptake by increasing the number of cell surface GLUT transporters. Evidences derived by cell culture experiments further supports the role of insulin-mediated PI3K activity in LA-induced glucose uptake, notably the sensitivity of this effect on wortmannin-a PI3K inhibitor $[14,85]$. Nevertheless, direct and relevant evidences of the role played by GLUT4 translocation to improve the glucose disposal via relevant administration of LA are still indispensable.

\section{Cancer}

Oxidative stress possesses also a main role in tumorigenesis [12]. LA has been administered as an anticancer agent mainly in experimental studies of different tumorigenesis cells type with encouraging results [2, 16, 23, 33, 41, 43]. Until now the molecular mechanisms implicated in this process are still unknown. In addition to its antioxidant power, another possibility could be its capability to provoke cellular apoptosis as recently demonstrated in lung cells $[43,63]$. This effects may originated by activation of caspase proteins induced by endoplasmic reticulum stress [41].

Another possible hypothesis could be linked to the cancer cells metabolism, which converts and transforms preferentially the glucose to lactate, a mechanism known as the Warburg effect [33]. In fact, LA is the cofactor of pyruvate dehydrogenase which converts and transforms the pyruvate molecules to acetyl CoA, resulting in a decrement in the formation of lactate molecules [16]. The clear and immediate consequence of this process is the block of the glycolytic way. In addition, the block of mTOR (target of rapamycin), a well known signaling pathway involved in cell growth and correlated to insulin receptor phosphorylation- PI3K-AKT activation, has been showed in several researches using insulinoma cells [72]. This action provoked an inhibition of insulin secretion as well as a decrement beta-cells growth [72].

Combinations of LA with well known drugs as well as the synthesis of LA conjugates with other bioactive scaf- folds are two strategies toward the development of effective agents for the prevention or treatment of various disorders and diseases. In agreement to the reviewed patents, LA can be used in combination therapy, with drugs (anticancer, antidiabetic, antimicrobial) having synergistic effects and reduced toxicity $[31,61]$. In fact, in some research studies, LA was administered associated with other antioxidant agents or with other anticancer drugs [10]. In this regard, some recent findings [74] well analyzed the effect of LA on neuroblastoma (NB) cells redox balance and how such antioxidant effect may impact on NB cells response to bortezomib (BTZ) drug. In particular, the data indicated that LA, in combination with BTZ, acted as chemical chaperone reducing the stress response induced by proteasome inhibition. Furthermore, these last results confirmed the neuroprotective effects of LA in neurological field, since it acts reducting both redox escalation and cellular damage induced by BTZ [74, 75].

\section{Concluding remarks}

Redox reactions belong to the major metabolic pathways of the cells. The shift of the balance between pro and antioxidants can account for mechanisms implicated in pathogenesis and/or progression of many, apparently unconnected pathological states, like tumors, neurodegenerative diseases, diabetes, etc. This underlines the importance of antioxidants in the therapy of these diseases. LA and DHLA have many biological functions involved in different intracellular systems resulting in a wide range of actions such as antioxidant protection, chelation of metal ions, regeneration of other antioxidant agents such as vitamin C, E and glutathione. Furthermore, LA/DHLA can also operate in multiple signaling transduction pathways, such as insulin, nuclear factor kappa B (NF-kB), nitric oxide synthesis and cellular apoptosis. Also, LA/DHLA can modulate directly or indirectly the expression of protein kinase $\mathrm{C}$ and AMPK that are both key enzymes in many downstream systems. It seems that LA is the most efficient drug of all antioxidants, which is confirmed by the following data:

- LA is administered orally since it is without difficulty absorbed in the stomach. LA goes through the bloodbrain barrier and does not show toxic effects and actions at doses used for prophylactic and therapeutic purposes.

- A lot of experimental and clinical studies proved beneficial effect of LA in such diseases as diabetes, atherosclerosis and heart diseases, cataract and neurodegenerative diseases. 
Fig. 3 Biological properties of LA: a LA mimic insulin stimulating glucose uptake. LA binds the tyrosine kinase domain inside the cell. LA can also modulate MAPK and NF$\mathrm{kB}$ activities, which may be independent of activation of receptor tyrosine kinases. b LA binds to $\mathrm{G}$ protein receptors, which in turn leads to activation of Adenyl cyclase (AC) able to generate cAMP from ATP. c LA exhibits many antioxidant properties: to restore endogenous antioxidants (recycle GSH); to protect from oxidative damage (scavenger activity of ROS and NOS); and metals chelation

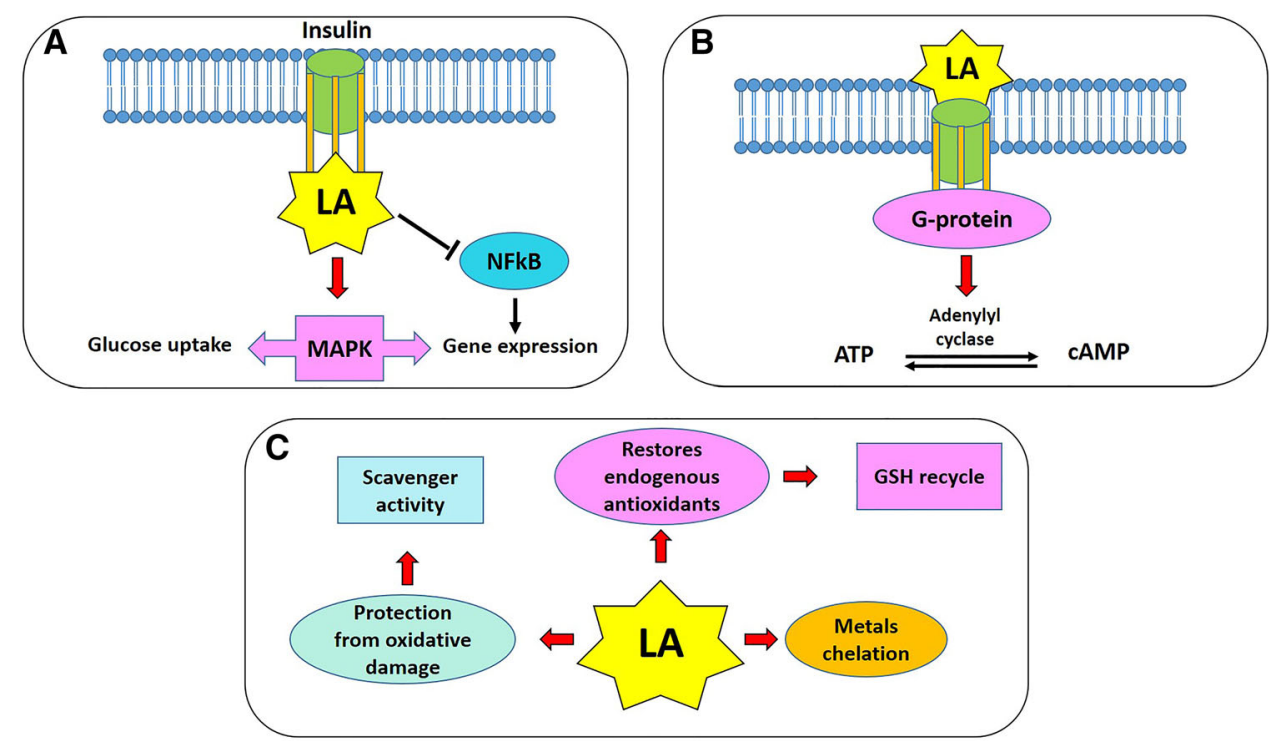

- Preliminary results on lipoic acid effect on alleviation of old age-related disorders and adverse influence of exercise are promising.

Furthermore, studies in vitro and in vivo demonstrated the wide ability of LA to influence biological functions (Fig. 3). In addition, as mentioned before, LA and DHLA both act as antioxidants to directly scavenge ROS and RNOS, chelate transition and heavy metal ions and mediate the recycling of other endogenous antioxidants as well as glutathione. LA also modulates various signaling cascades either by receptormediated or non-receptor-mediated processes.

Therefore, the beneficial properties of LA should not be doubted. However, further studies in the specific disease model systems are required to set proper dosage regimen as well as its involvement in cell growth and differentiation processes. Finally, the studies described herein provide promising data that can be used as a platform for future endeavors.

\section{Future perspectives}

Because LA performs a pleiotropic action on different pathways linked to the above mentioned diseases, its use as a potential therapeutic agent is very hopeful.

The activity of LA as a therapeutic agent is corroborated not only for its actions as a scavenger of ROS/RNS, but also for its capability to influence signaling cascades. The stimulation of signal transduction molecules containing critical cysteine residues may thus prove to be clinically useful and should be examined in detail in the next future.

One more significant remark for future researches is the differential outcome of LA administered in vitro and in vivo. There is still much to learn regarding to the metabolic destiny of LA and the effects of the various metabolites on the cells. In fact, it is obvious that LA is quickly removed from plasma and tissues, whereas (DH)LA may persist within cell culture media for long time. This underlines the importance of using in vivo models to confirm the data obtained in vitro.

Many preclinical efforts on LA should be conducted keeping in mind these considerations. Finally, this review had the objective to extend the biochemical and biological knowledge about LA, even if it is clear that we still need more knowledge to study in deep about this multifunctional compound in preclinical phase, before to offer and extend its use in routine clinical practice.

Acknowledgements The authors acknowledged pharmaceutical MDM S.p.A. Via Volturno, 29/b-20900 Monza (MB), Italy, e-mail: mdm@mdmspa.com.

Author contributions All authors had full access to all the data in the study and take responsibility for the integrity of the data and the accuracy of the data analysis. Study concept and design: RA and VB. Managing the literature searches: DT, SG, CG, DT. Drafting of the manuscript: RA and VB. Critical revision of the manuscript: DT, CG, CDA, GL, GLV, FA, RA and VB. Administrative and technical support: CG, DT. Supervision of the study: DT, RA and VB. Approving the final draft of manuscript: all the authors.

\section{Compliance with ethical standards}

Conflict of interest All authors declare no conflict of interest.

\section{References}

1. Abdul HM, Butterfield DA. Involvement of PI3K/PKG/ERK1/2 signaling pathways in cortical neurons to trigger protection by cotreatment of acetyl-L-carnitine and alpha-lipoic acid against HNE-mediated oxidative stress and neurotoxicity: implications for Alzheimer's disease. Free Radic Biol Med. 2007;42(3): $371-84$. 
2. Al Abdan M. Alfa-lipoic acid controls tumor growth and modulates hepatic redox state in Ehrlich-ascites-carcinoma-bearing mice. Sci World J. 2012;2012:509838.

3. Ametov AS, Barinov A, Dyck PJ, Hermann R, Kozlova N, Litchy WJ, Low PA, Nehrdich D, Novosadova M, O’Brien PC, Reljanovic M, Samigullin R, Schuette K, Strokov I, Tritschler HJ, Wessel K, Yakhno N, Ziegler D, S. T. S. Group. The sensory symptoms of diabetic polyneuropathy are improved with alpha-lipoic acid: the SYDNEY trial. Diabetes Care. 2003;26(3):770-776.

4. Bierhaus A, Chevion S, Chevion M, Hofmann M, Quehenberger $\mathrm{P}$, Illmer T, Luther T, Berentshtein E, Tritschler H, Muller M, Wahl P, Ziegler R, Nawroth PP. Advanced glycation end product-induced activation of NF-kappaB is suppressed by alphalipoic acid in cultured endothelial cells. Diabetes. 1997;46(9):1481-90.

5. Bo L, Dawson TM, Wesselingh S, Mork S, Choi S, Kong PA, Hanley D, Trapp BD. Induction of nitric oxide synthase in demyelinating regions of multiple sclerosis brains. Ann Neurol. 1994;36(5):778-86.

6. Bramanti V, Tomassoni D, Bronzi D, Grasso S, Curro M, Avitabile M, Li Volsi G, Renis M, Ientile R, Amenta F, Avola R. Alpha-lipoic acid modulates GFAP, vimentin, nestin, cyclin D1 and MAP-kinase expression in astroglial cell cultures. Neurochem Res. 2010;35(12):2070-7.

7. Chang MY, Gwon TM, Lee HS, Lee JH, Oh SH, Kim SJ, Park $\mathrm{MH}$. The effect of systemic lipoic acid on hearing preservation after cochlear implantation via the round window approach: a guinea pig model. Eur J Pharmacol. 2017;799:67-72.

8. Cho KJ, Moon HE, Moini H, Packer L, Yoon DY, Chung AS. Alpha-lipoic acid inhibits adipocyte differentiation by regulating pro-adipogenic transcription factors via mitogen-activated protein kinase pathways. J Biol Chem. 2003;278(37):34823-33.

9. Cromheeke KM, Kockx MM, De Meyer GR, Bosmans JM, Bult H, Beelaerts WJ, Vrints CJ, Herman AG. Inducible nitric oxide synthase colocalizes with signs of lipid oxidation/peroxidation in human atherosclerotic plaques. Cardiovasc Res. 1999;43(3):744-54.

10. Diesel B, Kulhanek-Heinze S, Holtje M, Brandt B, Holtje HD, Vollmar AM, Kiemer AK. Alpha-lipoic acid as a directly binding activator of the insulin receptor: protection from hepatocyte apoptosis. Biochemistry. 2007;46(8):2146-55.

11. Duby JJ, Campbell RK, Setter SM, White JR, Rasmussen KA. Diabetic neuropathy: an intensive review. Am J Health Syst Pharm. 2004;61(2):160-173 (quiz 175-166).

12. Durand M, Mach N. Alpha lipoic acid and its antioxidant against cancer and diseases of central sensitization. Nutr Hosp. 2013;28(4):1031-8.

13. El-Osta A, Brasacchio D, Yao D, Pocai A, Jones PL, Roeder RG, Cooper ME, Brownlee M. Transient high glucose causes persistent epigenetic changes and altered gene expression during subsequent normoglycemia. J Exp Med. 2008;205(10):2409-17.

14. Estrada DE, Ewart HS, Tsakiridis T, Volchuk A, Ramlal T, Tritschler H, Klip A. Stimulation of glucose uptake by the natural coenzyme alpha-lipoic acid/thioctic acid: participation of elements of the insulin signaling pathway. Diabetes. 1996;45(12):1798-804.

15. Evans JL, Goldfine ID. Alpha-lipoic acid: a multifunctional antioxidant that improves insulin sensitivity in patients with type 2 diabetes. Diabetes Technol Ther. 2000;2(3):401-13.

16. Feuerecker B, Pirsig S, Seidl C, Aichler M, Feuchtinger A, Bruchelt G, Senekowitsch-Schmidtke R. Lipoic acid inhibits cell proliferation of tumor cells in vitro and in vivo. Cancer Biol Ther. 2012;13(14):1425-35.

17. Gasic-Milenkovic J, Loske C, Munch G. Advanced glycation endproducts cause lipid peroxidation in the human neuronal cell line SH-SY5Y. J Alzheimers Dis. 2003;5(1):25-30.
18. Gerthoffer WT, Singer CA. MAPK regulation of gene expression in airway smooth muscle. Respir Physiol Neurobiol. 2003;137(2-3):237-50.

19. Ghibu S, Richard C, Vergely C, Zeller M, Cottin Y, Rochette L. Antioxidant properties of an endogenous thiol: alpha-lipoic acid, useful in the prevention of cardiovascular diseases. J Cardiovasc Pharmacol. 2009;54(5):391-8.

20. Golbidi S, Badran M, Laher I. Diabetes and alpha lipoic acid. Front Pharmacol. 2011;2:69.

21. Gomes MB, Negrato CA. Alpha-lipoic acid as a pleiotropic compound with potential therapeutic use in diabetes and other chronic diseases. Diabetol Metab Syndr. 2014;6(1):80.

22. Grasso S, Bramanti V, Tomassoni D, Bronzi D, Malfa G, Traini E, Napoli M, Renis M, Amenta F, Avola R. Effect of lipoic acid and alpha-glyceryl-phosphoryl-choline on astroglial cell proliferation and differentiation in primary culture. J Neurosci Res. 2014;92(1):86-94.

23. Guais A, Baronzio G, Sanders E, Campion F, Mainini C, Fiorentini G, Montagnani F, Behzadi M, Schwartz L, Abolhassani M. Adding a combination of hydroxycitrate and lipoic acid (METABLOC) to chemotherapy improves effectiveness against tumor development: experimental results and case report. Invest New Drugs. 2012;30(1):200-11.

24. Hagen TM, Ingersoll RT, Lykkesfeldt J, Liu J, Wehr CM, Vinarsky V, Bartholomew JC, Ames AB. (R)-alpha-lipoic acidsupplemented old rats have improved mitochondrial function, decreased oxidative damage, and increased metabolic rate. FASEB J. 1999;13(2):411-8.

25. Hedges JC, Singer CA, Gerthoffer WT. Mitogen-activated protein kinases regulate cytokine gene expression in human airway myocytes. Am J Respir Cell Mol Biol. 2000;23(1):86-94.

26. Henriksen EJ, Jacob S, Streeper RS, Fogt DL, Hokama JY, Tritschler HJ. Stimulation by alpha-lipoic acid of glucose transport activity in skeletal muscle of lean and obese Zucker rats. Life Sci. 1997;61(8):805-12.

27. Hiller S, DeKroon R, Hamlett ED, Xu L, Osorio C, Robinette J, Winnik W, Simington S, Maeda N, Alzate O, Yi X. Alpha-lipoic acid supplementation protects enzymes from damage by nitrosative and oxidative stress. Biochim Biophys Acta. 2016;1860(1 Pt A):36-45.

28. Holmquist L, Stuchbury G, Berbaum K, Muscat S, Young S, Hager K, Engel J, Munch G. Lipoic acid as a novel treatment for Alzheimer's disease and related dementias. Pharmacol Ther. 2007;113(1):154-64.

29. Jacob S, Henriksen EJ, Tritschler HJ, Augustin HJ, Dietze GJ. Improvement of insulin-stimulated glucose-disposal in type 2 diabetes after repeated parenteral administration of thioctic acid. Exp Clin Endocrinol Diabetes. 1996;104(3):284-8.

30. Jacob S, Ruus P, Hermann R, Tritschler HJ, Maerker E, Renn W, Augustin HJ, Dietze GJ, Rett K. Oral administration of RACalpha-lipoic acid modulates insulin sensitivity in patients with type-2 diabetes mellitus: a placebo-controlled pilot trial. Free Radic Biol Med. 1999;27(3-4):309-14.

31. Kates SA, Casale RA, Baguisi A, Beeuwkes R 3rd. Lipoic acid analogs with enhanced pharmacological activity. Bioorg Med Chem. 2014;22(1):505-12.

32. Khanna S, Roy S, Packer L, Sen CK. Cytokine-induced glucose uptake in skeletal muscle: redox regulation and the role of alphalipoic acid. Am J Physiol. 1999;276(5 Pt 2):R1327-33.

33. Kim JI, Cho SR, Lee CM, Park ES, Kim KN, Kim HC, Lee HY. Induction of ER stress-mediated apoptosis by alpha-lipoic acid in A549 cell lines. Korean J Thorac Cardiovasc Surg. 2012;45(1):1-10.

34. Kim MS, Park JY, Namkoong C, Jang PG, Ryu JW, Song HS, Yun JY, Namgoong IS, Ha J, Park IS, Lee IK, Viollet B, Youn JH, Lee HK, Lee KU. Anti-obesity effects of alpha-lipoic acid 
mediated by suppression of hypothalamic AMP-activated protein kinase. Nat Med. 2004;10(7):727-33.

35. Koh G, Yang EJ, Kim MK, Lee SA, Lee DH. Alpha-lipoic acid treatment reverses 2-deoxy-D-ribose-induced oxidative damage and suppression of insulin expression in pancreatic beta-cells. Biol Pharm Bull. 2013;36(10):1570-6.

36. Koprowski H, Zheng YM, Heber-Katz E, Fraser N, Rorke L, Fu $\mathrm{ZF}$, Hanlon C, Dietzschold B. In vivo expression of inducible nitric oxide synthase in experimentally induced neurologic diseases. Proc Natl Acad Sci USA. 1993;90(7):3024-7.

37. Kwiecien B, Dudek M, Bilska-Wilkosz A, Knutelska J, Bednarski M, Kwiecien I, Zygmunt M, Iciek M, SokolowskaJezewicz M, Sapa J, Wlodek L. In vivo anti-inflammatory activity of lipoic acid derivatives in mice. Postepy Hig Med Dosw (Online). 2013;67:331-8.

38. Li Volti G, Salomone S, Sorrenti V, Mangiameli A, Urso V, Siarkos I, Galvano F, Salamone F. Effect of silibinin on endothelial dysfunction and ADMA levels in obese diabetic mice. Cardiovasc Diabetol. 2011;10:62.

39. Lovell MA, Markesbery WR. Oxidative damage in mild cognitive impairment and early Alzheimer's disease. J Neurosci Res. 2007;85(14):3036-40.

40. Lovell MA, Xie C, Xiong S, Markesbery WR. Protection against amyloid beta peptide and iron/hydrogen peroxide toxicity by alpha lipoic acid. J Alzheimers Dis. 2003;5(3):229-39.

41. Mantovani G, Maccio A, Madeddu C, Mura L, Gramignano G, Lusso MR, Murgia V, Camboni P, Ferreli L, Mocci M, Massa E. The impact of different antioxidant agents alone or in combination on reactive oxygen species, antioxidant enzymes and cytokines in a series of advanced cancer patients at different sites: correlation with disease progression. Free Radic Res. 2003;37(2):213-23.

42. Merz PA, Wisniewski HM, Somerville RA, Bobin SA, Masters CL, Iqbal K. Ultrastructural morphology of amyloid fibrils from neuritic and amyloid plaques. Acta Neuropathol. 1983;60(1-2):113-24.

43. Michikoshi H, Nakamura T, Sakai K, Suzuki Y, Adachi E, Matsugo S, Matsumoto K. alpha-Lipoic acid-induced inhibition of proliferation and met phosphorylation in human non-small cell lung cancer cells. Cancer Lett. 2013;335(2):472-8.

44. Moini H, Tirosh O, Park YC, Cho KJ, Packer L. R-alpha-lipoic acid action on cell redox status, the insulin receptor, and glucose uptake in 3T3-L1 adipocytes. Arch Biochem Biophys. 2002;397(2):384-91.

45. Moura FA, de Andrade KQ, dos Santos JC, Goulart MO. Lipoic Acid: its antioxidant and anti-inflammatory role and clinical applications. Curr Top Med Chem. 2015;15(5):458-83.

46. Muller U, Krieglstein J. Prolonged pretreatment with alpha-lipoic acid protects cultured neurons against hypoxic, glutamate-, or iron-induced injury. J Cereb Blood Flow Metab. 1995;15(4):624-30.

47. Nagamatsu M, Nickander KK, Schmelzer JD, Raya A, Wittrock DA, Tritschler H, Low PA. Lipoic acid improves nerve blood flow, reduces oxidative stress, and improves distal nerve conduction in experimental diabetic neuropathy. Diabetes Care. 1995;18(8):1160-7.

48. Newsholme P, Rebelato E, Abdulkader F, Krause M, Carpinelli A, Curi R. Reactive oxygen and nitrogen species generation, antioxidant defenses, and beta-cell function: a critical role for amino acids. J Endocrinol. 2012;214(1):11-20.

49. Nickander KK, McPhee BR, Low PA, Tritschler H. Alpha-lipoic acid: antioxidant potency against lipid peroxidation of neural tissues in vitro and implications for diabetic neuropathy. Free Radic Biol Med. 1996;21(5):631-9.

50. Ou P, Tritschler HJ, Wolff SP. Thioctic (lipoic) acid: a therapeutic metal-chelating antioxidant? Biochem Pharmacol. 1995;50(1):123-6.
51. Packer L. alpha-Lipoic acid: a metabolic antioxidant which regulates NF-kappa B signal transduction and protects against oxidative injury. Drug Metab Rev. 1998;30(2):245-75.

52. Packer L, Kraemer K, Rimbach G. Molecular aspects of lipoic acid in the prevention of diabetes complications. Nutrition. 2001;17(10):888-95.

53. Packer L, Tritschler HJ, Wessel K. Neuroprotection by the metabolic antioxidant alpha-lipoic acid. Free Radic Biol Med. 1997;22(1-2):359-78.

54. Palmer RM, Smith RE. Commentary on viewpoint article by $\mathrm{AH}$ Henderson, MJ Lewis, AM Shah, and JA Smith (April, pp 305-308). Cardiovasc Res. 1992;26(6):638.

55. Patrick L. Mercury toxicity and antioxidants: part 1: role of glutathione and alpha-lipoic acid in the treatment of mercury toxicity. Altern Med Rev. 2002;7(6):456-71.

56. Perez-Matos MC, Morales-Alvarez MC, Mendivil CO. Lipids: a suitable therapeutic target in diabetic neuropathy? J Diabetes Res. 2017;2017:6943851.

57. Pick U, Haramaki N, Constantinescu A, Handelman GJ, Tritschler HJ, Packer L. Glutathione reductase and lipoamide dehydrogenase have opposite stereospecificities for alpha-lipoic acid enantiomers. Biochem Biophys Res Commun. 1995;206(2):724-30.

58. Quinn JF, Bussiere JR, Hammond RS, Montine TJ, Henson E, Jones RE, Stackman RW Jr. Chronic dietary alpha-lipoic acid reduces deficits in hippocampal memory of aged $\mathrm{Tg} 2576$ mice. Neurobiol Aging. 2007;28(2):213-25.

59. Ramamurthy S, Ronnett G. AMP-activated protein kinase (AMPK) and energy-sensing in the brain. Exp Neurobiol. 2012;21(2):52-60.

60. Roman-Pintos LM, Villegas-Rivera G, Rodriguez-Carrizalez AD, Miranda-Diaz AG, Cardona-Munoz EG. Diabetic polyneuropathy in type 2 Diabetes Mellitus: inflammation, oxidative stress, and mitochondrial function. J Diabetes Res. 2016;2016:3425617.

61. Sacerdoti D, Colombrita C, Ghattas MH, Ismaeil EF, Scapagnini G, Bolognesi M, Li Volti G, Abraham NG. Heme oxygenase-1 transduction in endothelial cells causes downregulation of monocyte chemoattractant protein-1 and of genes involved in inflammation and growth. Cell Mol Biol (Noisy-le-grand). 2005;51(4):363-370.

62. Salinthone S, Yadav V, Bourdette DN, Carr DW. Lipoic acid: a novel therapeutic approach for multiple sclerosis and other chronic inflammatory diseases of the CNS. Endocr Metab Immune Disord Drug Targets. 2008;8(2):132-42.

63. Salomone F, Barbagallo I, Puzzo L, Piazza C, Li Volti G. Efficacy of adipose tissue-mesenchymal stem cell transplantation in rats with acetaminophen liver injury. Stem Cell Res. 2013;11(3):1037-44.

64. Shaw JE, Zimmet PZ, de Courten M, Dowse GK, Chitson P, Gareeboo H, Hemraj F, Fareed D, Tuomilehto J, Alberti KG. Impaired fasting glucose or impaired glucose tolerance. What best predicts future diabetes in Mauritius? Diabetes Care. 1999;22(3):399-402.

65. Shen QW, Zhu MJ, Tong J, Ren J, Du M. Ca2+/calmodulindependent protein kinase is involved in AMP-activated protein kinase activation by alpha-lipoic acid in $\mathrm{C} 2 \mathrm{C} 12$ myotubes. Am J Physiol Cell Physiol. 2007;293(4):C1395-403.

66. Singer CA, Baker KJ, McCaffrey A, AuCoin DP, Dechert MA, Gerthoffer WT. p38 MAPK and NF-kappaB mediate COX-2 expression in human airway myocytes. Am J Physiol Lung Cell Mol Physiol. 2003;285(5):L1087-98.

67. Smith AR, Shenvi SV, Widlansky M, Suh JH, Hagen TM. Lipoic acid as a potential therapy for chronic diseases associated with oxidative stress. Curr Med Chem. 2004;11(9):1135-46.

68. Smith AR, Visioli F, Frei B, Hagen TM. Lipoic acid significantly restores, in rats, the age-related decline in vasomotion. $\mathrm{Br} \mathrm{J}$ Pharmacol. 2008;153(8):1615-22. 
69. Steinberg GR, Kemp BE. AMPK in health and disease. Physiol Rev. 2009;89(3):1025-78.

70. Suh JH, Moreau R, Heath SH, Hagen TM. Dietary supplementation with (R)-alpha-lipoic acid reverses the age-related accumulation of iron and depletion of antioxidants in the rat cerebral cortex. Redox Rep. 2005;10(1):52-60.

71. Suh JH, Zhu BZ, deSzoeke E, Frei B, Hagen TM. Dihydrolipoic acid lowers the redox activity of transition metal ions but does not remove them from the active site of enzymes. Redox Rep. 2004;9(1):57-61.

72. Targonsky ED, Dai F, Koshkin V, Karaman GT, Gyulkhandanyan AV, Zhang Y, Chan CB, Wheeler MB. alpha-lipoic acid regulates AMP-activated protein kinase and inhibits insulin secretion from beta cells. Diabetologia. 2006;49(7):1587-98.

73. Tibullo D, Barbagallo I, Giallongo C, La Cava P, Parrinello N, Vanella L, Stagno F, Palumbo GA, Li Volti G, Di Raimondo F. Nuclear translocation of heme oxygenase-1 confers resistance to imatinib in chronic myeloid leukemia cells. Curr Pharm Des. 2013;19(15):2765-70.

74. Tibullo D, Giallongo C, Puglisi F, Tomassoni D, Camiolo G, Cristaldi M, Brundo MV, Anfuso CD, Lupo G, Stampone T, Li Volti G, Amenta F, Avola R, Bramanti V. Effect of lipoic acid on the biochemical mechanisms of resistance to Bortezomib in SHSY5Y Neuroblastoma Cells. Mol Neurobiol. 2017.

75. Torella D, Leosco D, Indolfi C, Curcio A, Coppola C, Ellison GM, Russo VG, Torella M, Li Volti G, Rengo F, Chiariello M. Aging exacerbates negative remodeling and impairs endothelial regeneration after balloon injury. Am J Physiol Heart Circ Physiol. 2004;287(6):H2850-60.

76. Vallianou N, Evangelopoulos A, Koutalas P. Alpha-lipoic acid and diabetic neuropathy. Rev Diabet Stud. 2009;6(4):230-6.

77. van der Goes A, Brouwer J, Hoekstra K, Roos D, van den Berg TK, Dijkstra CD. Reactive oxygen species are required for the phagocytosis of myelin by macrophages. J Neuroimmunol. 1998;92(1-2):67-75.

78. Vinik AI, Park TS, Stansberry KB, Pittenger GL. Diabetic neuropathies. Diabetologia. 2000;43(8):957-73.

79. Wang Y, Li X, Guo Y, Chan L, Guan X. alpha-Lipoic acid increases energy expenditure by enhancing adenosine monophosphate-activated protein kinase-peroxisome proliferatoractivated receptor-gamma coactivator-1alpha signaling in the skeletal muscle of aged mice. Metabolism. 2010;59(7):967-76.

80. Wenk GL. Neuropathologic changes in Alzheimer's disease: potential targets for treatment. J Clin Psychiatry. 2006;67(Suppl 3):3-7 (quiz 23).
81. Winiarska K, Malinska D, Szymanski K, Dudziak M, Bryla J. Lipoic acid ameliorates oxidative stress and renal injury in alloxan diabetic rabbits. Biochimie. 2008;90(3):450-9.

82. Wong WS. Inhibitors of the tyrosine kinase signaling cascade for asthma. Curr Opin Pharmacol. 2005;5(3):264-71.

83. Xing ZG, Yu GD, Qin L, Jiang F, Zhao WH. Effects and mechanism of lipoic acid on beta-amyloid-intoxicated C6 glioma cells. Genet Mol Res. 2015;14(4):13880-8.

84. Yamamoto Y, Gaynor RB. Therapeutic potential of inhibition of the NF-kappaB pathway in the treatment of inflammation and cancer. J Clin Invest. 2001;107(2):135-42.

85. Yaworsky K, Somwar R, Ramlal T, Tritschler HJ, Klip A. Engagement of the insulin-sensitive pathway in the stimulation of glucose transport by alpha-lipoic acid in 3T3-L1 adipocytes. Diabetologia. 2000;43(3):294-303.

86. Ying Z, Kampfrath T, Sun Q, Parthasarathy S, Rajagopalan S. Evidence that alpha-lipoic acid inhibits NF-kappaB activation independent of its antioxidant function. Inflamm Res. 2011;60(3):219-25.

87. Zhang J, McCullough PA. Lipoic acid in the prevention of acute kidney injury. Nephron. 2016;134(3):133-40.

88. Zhou G, Myers R, Li Y, Chen Y, Shen X, Fenyk-Melody J, Wu M, Ventre J, Doebber T, Fujii N, Musi N, Hirshman MF, Goodyear LJ, Moller DE. Role of AMP-activated protein kinase in mechanism of metformin action. $\mathrm{J}$ Clin Invest. 2001;108(8):1167-74.

89. Ziegler D, Ametov A, Barinov A, Dyck PJ, Gurieva I, Low PA, Munzel U, Yakhno N, Raz I, Novosadova M, Maus J, Samigullin R. Oral treatment with alpha-lipoic acid improves symptomatic diabetic polyneuropathy: the SYDNEY 2 trial. Diabetes Care. 2006;29(11):2365-70.

90. Ziegler D, Hanefeld M, Ruhnau KJ, Hasche H, Lobisch M, Schutte K, Kerum G, Malessa R. Treatment of symptomatic diabetic polyneuropathy with the antioxidant alpha-lipoic acid: a 7-month multicenter randomized controlled trial (ALADIN III study). ALADIN III Study Group. Alpha-Lipoic Acid in Diabetic Neuropathy. Diabetes Care. 1999;22(8):1296-301.

91. Ziegler D, Nowak H, Kempler P, Vargha P, Low PA. Treatment of symptomatic diabetic polyneuropathy with the antioxidant alpha-lipoic acid: a meta-analysis. Diabet Med. 2004;21(2):114-21. 